Research Paper

\title{
Diagnostic Accuracy of the Neck Tornado Test as a New Screening Test in Cervical Radiculopathy
}

Juyeon Park ${ }^{1}$, Woo Young Park2,3, Seungbae Hong4, Jiwon An, Jae Chul Koh ${ }^{6}$, Youn-Woo Lee ${ }^{5}$, Yong Chan $\mathrm{Kim}^{7}$, Jong Bum Choi ${ }^{4}$

1. Department of Anesthesia and Pain Medicine, Pusan National University Yangsan Hospital, Pusan, Korea;

2. Department of Anesthesia, Sheikh Khalifa Specialty Hospital, Ras Al Khaimah, United Arab Emirates;

3. Department of Anesthesiology and Pain Medicine, Seoul National University College of Medicine, Seoul, Korea;

4. Department of Anesthesiology and Pain Medicine, Ajou University College of Medicine, Suwon, Korea;

5. Department of Anesthesiology and Pain Medicine, Yonsei University College of Medicine, Gangnam Severance Hospital, Seoul, Korea;

6. Department of Anesthesiology and Pain Medicine, Korea University College of Medicine, Korea University Anam Hospital, Seoul, Korea;

7. Department of Anesthesiology and Pain Medicine, Gwangmyeong Saeum Hospital, Gwangmyeong, Korea.

$\triangle$ Corresponding author: Dr. Jong Bum Choi, Department of Anesthesiology and Pain Medicine, Ajou University College of Medicine, 164 World Cup-ro, Yeongtong-gu, Suwon 16499, Korea Telephone: +82-31-219-5571 Fax: +82-31-219-5579 E-mail: romeojb@naver.com

(C) Ivyspring International Publisher. This is an open access article distributed under the terms of the Creative Commons Attribution (CC BY-NC) license (https://creativecommons.org/licenses/by-nc/4.0/). See http://ivyspring.com/terms for full terms and conditions.

Received: 2017.01.08; Accepted: 2017.04.17; Published: 2017.06.23

\begin{abstract}
Background: The Spurling test, although a highly specific provocative test of the cervical spine in cervical radiculopathy (CR), has low to moderate sensitivity. Thus, we introduced the neck tornado test (NTT) to examine the neck and the cervical spine in CR.

Objectives: The aim of this study was to introduce a new provocative test, the NTT, and compare the diagnostic accuracy with a widely accepted provocative test, the Spurling test.

Design: Retrospective study.

Methods: Medical records of 135 subjects with neck pain (CR, $n=67$; without $C R, n=68)$ who had undergone cervical spine magnetic resonance imaging and been referred to the pain clinic between September 2014 and August 2015 were reviewed. Both the Spurling test and NTT were performed in all patients by expert examiners. Sensitivity, specificity, and accuracy were compared for both the Spurling test and the NTT.

Results: The sensitivity of the Spurling test and the NTT was $55.22 \%$ and $85.07 \%(P<0.0001)$; specificity, $98.53 \%$ and $86.76 \%(P=0.0026)$; accuracy, $77.04 \%$ and $85.93 \%(P=0.0423)$, respectively.

Conclusions: The NTT is more sensitive with superior diagnostic accuracy for CR diagnosed by magnetic resonance imaging than the Spurling test.
\end{abstract}

Key words: cervical radiculopathy, neck pain, radicular pain, Spurling test, neck tornado test.

\section{Introduction}

Cervical radiculopathy $(\mathrm{CR})$ is defined as pain in a radicular pattern in one or both upper extremities related to compression and/or irritation of one or more cervical nerve roots [1] and is commonly caused by posterolateral herniation of a cervical disc, degeneration of a disc causing decreased height of the neural foramen, and cervical spondylosis. When pain radiates in the arm and is associated with sensory and motor disturbances, $\mathrm{CR}$ is suspected [2]. $\mathrm{CR}$ is a common diagnosis, with an age-adjusted incidence of 83 cases per 100,000 persons [3].

When patients with neck or arm pain visit a pain clinic, physical examinations are performed to screen $\mathrm{CR}$ before spine MRI or CT because MRI is expensive and $\mathrm{CT}$ scan has radiation hazard. A number of provocative tests and signs described in the literature can be used as a screening test of CR [4] before using spine MRI or CT, and the Spurling test is one of them 
(Figure 1). However, despite its high specificity $(92 \%-100 \%)$, its sensitivity is only low to moderate $(30 \%-60 \%)$, depending on the study [5, 6-10]. We hypothesized that the low to moderate sensitivity was because the compressive force used to exacerbate the encroachment of the exiting nerve roots was only applied at a single direction (Figure 2) [6]. Thus, we designed a new physical examination that would apply compressive force at all angles by rotating the neck in a $180^{\circ}$ tornadic pattern while pressure is applied, the neck tornado test (NTT) (also denominated as "Choi's test" after the name of the originator, Dr. Jong Bum Choi) (Figure 3 and 4).
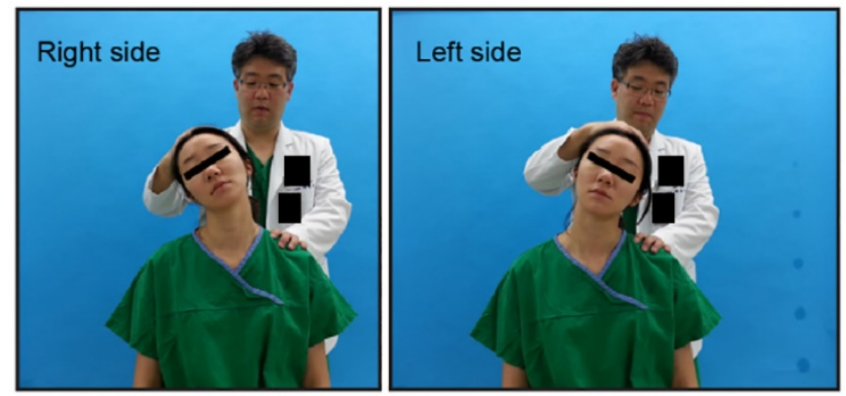

Figure 1. The Spurling test. Neck extension and lateral bending were performed.

A
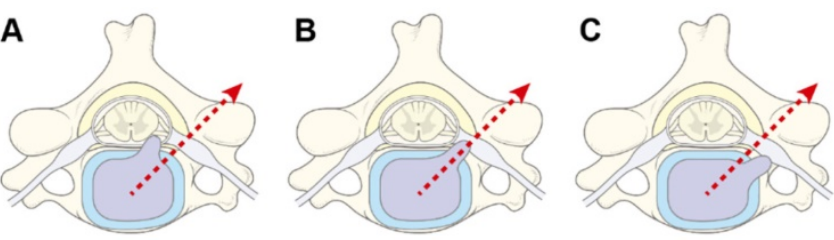

Figure 2. The force to exacerbate the encroachment of the exiting nerve roots was only applied at a single direction. The Spurling test was negative in (A) and (C) and positive in (B).
The aim of this study was to introduce a new provocative test, the NTT, examine the neck and the cervical spine in $\mathrm{CR}$, and compare the diagnostic accuracy with a widely accepted provocative test, the Spurling test.

\section{Methods}

This research introduced the cervical spine examination, the NTT. To evaluate the usefulness of the NTT, its sensitivity, specificity, PPV, NPV, and accuracy were compared with those of the Spurling test. This retrospective study was approved by the Institutional Review Board and the ethics committee of our hospital (Approval No. 3-2015-0260).

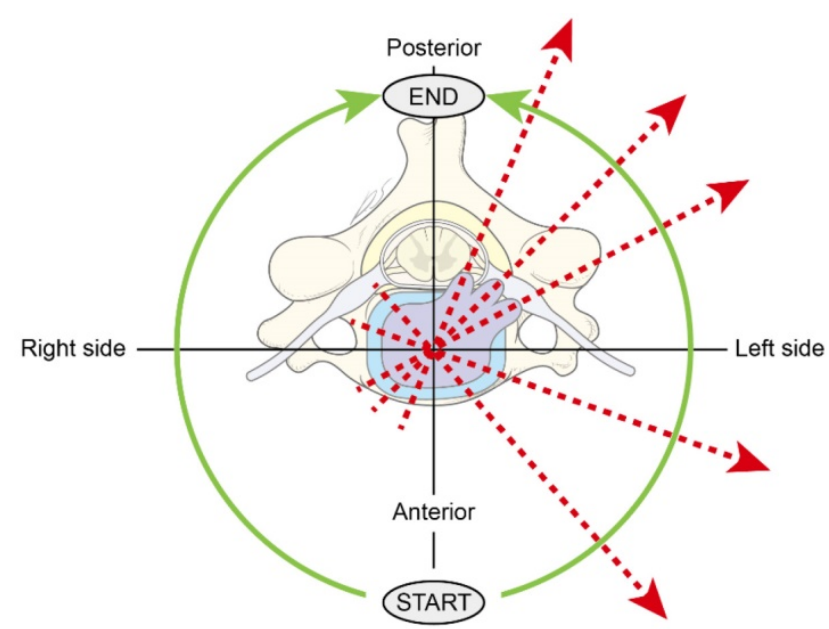

Figure 3. Force is applied at all angles by rotating the neck in a $180^{\circ}$ tornadic pattern.

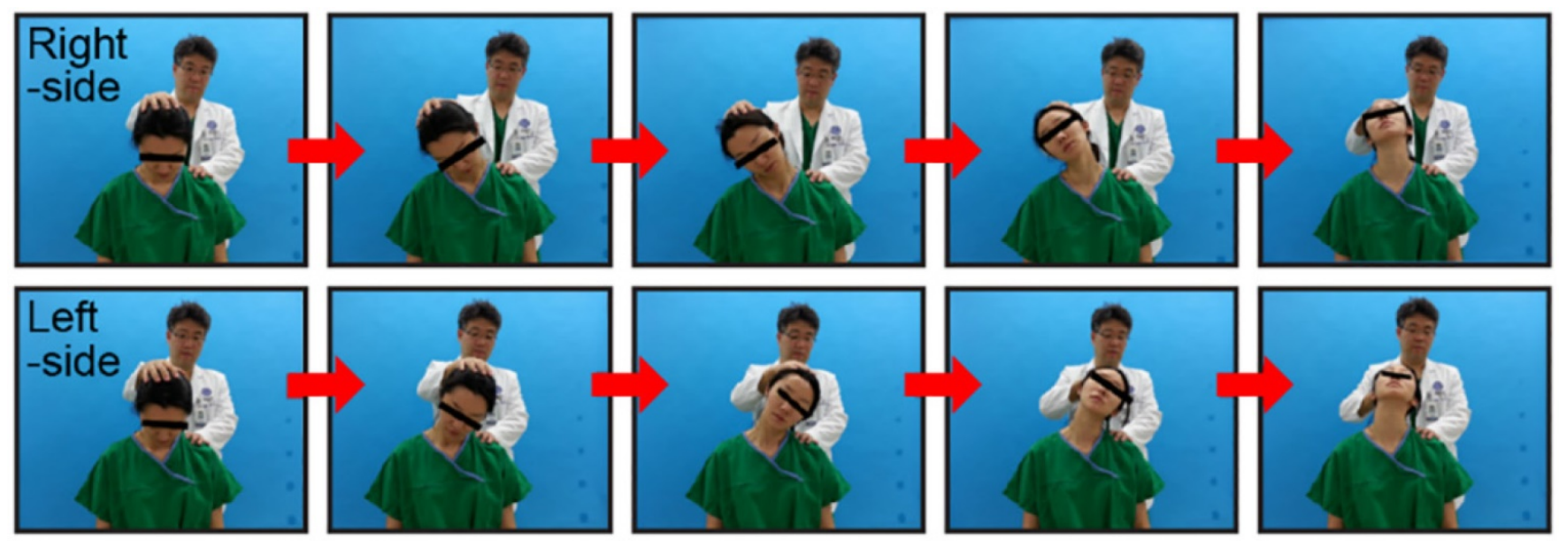

Figure 4. The neck tornado test (Choi's test). 


\section{Patients}

Medical records of 135 subjects with neck pain who had undergone cervical spine MRI and been referred to the pain clinic of our hospital between September 2014 and August 2015 were reviewed. Demographic data are given in Table 1. CR was defined as the expression of typical symptoms of CR such as arm pain, neck pain, scapular or periscapular pain, paresthesia, numbness, sensory change, weakness, or sign of abnormal deep tendon reflex in the arm, with cervical disc herniation or other lesions that decrease the dimensions of the foramen on MRI studies [5]. CR was confirmed by a pain clinician with a 10-year experience by considering the MRI findings and CR symptoms after the Spurling test and the NTT. Patients with neck pain who had undergone cervical spine MRI were included in the study. The exclusion criteria were pregnancy and/or history of cervical spine surgery, inflammatory disease such as rheumatoid arthritis, or previous nerve block for CR.

Table 1. Demographic data.

\begin{tabular}{lllll}
\hline & $\begin{array}{l}\text { Patients } \\
(\mathrm{n}=135)\end{array}$ & $\begin{array}{l}\text { With CR } \\
(\mathrm{n}=67)\end{array}$ & $\begin{array}{l}\text { Without CR } \\
(\mathrm{n}=68)\end{array}$ & $P$ value \\
\hline Age, $\mathrm{y}$ & $53.4 \pm 13.1$ & $52.1 \pm 12.7$ & $54.7 \pm 13.6$ & 0.2517 \\
Weight, $\mathrm{kg}$ & $66.4 \pm 14.2$ & $67.8 \pm 16.9$ & $65.0 \pm 11.3$ & 0.2830 \\
Height, $\mathrm{cm}$ & $165.3 \pm 8.3$ & $166.1 \pm 7.6$ & $164.6 \pm 8.9$ & 0.3152 \\
Male/female & $78 / 57$ & $41 / 26$ & $36 / 32$ & 0.3364 \\
\hline
\end{tabular}

CR: cervical radiculopathy.

\section{Spurling test}

Physical examinations were performed on all patients. One examiner examined the consecutive 135 subjects. The Spurling test was performed during history taking and examination, 10 minutes before the NTT, and was initiated by neck extension, rotation, and downward pressure on the head [11].

\section{Neck tornado test (Choi's test)}

The NTT was performed in the same position, with the patient seated and the same examiner standing behind the patient, as in the Spurling test. Light vertical pressure is applied by the examiner's one hand on the patient's vertex while the neck is relaxed. The body of patient is fixed by the other hand and cannot be influenced by the NTT. During the NTT, light vertical pressure is applied to the cervical spine continuously. Rotation of the neck is initiated, with the patient's neck flexed maximally from the vertical axis. The neck is rotated toward the direction of the pain site, progressing to full lateral bending and full extension of $180^{\circ}$, in a tornadic pattern (Figure 3). The results of both the Spurling test and the NTT were scored as either positive or negative, with reproduced or aggravated radicular pain or tingling in the ipsilateral shoulder or upper extremity indicating a positive sign [7]. Reproduced or aggravated signs in the contralateral side of the physical examination were ignored and recorded as negative.

\section{Statistical analyses}

Sensitivity (true positive/[true positive + false negative]), specificity (true negative/[true negative + false positive]), accuracy ([true positive + true negative]/total patients), positive predictive value (PPV; true positive/[true positive + false positive]), and negative predictive value (NPV; true negative/[true negative + false negative]) were calculated and compared for both the Spurling test and the NTT. The diagnostic performance of the Spurling test and the NTT was compared via generalized estimating equation using SAS version 9 . 2 (SAS Institute, Cary, NC, USA) and assessed by receiver operating characteristic (ROC) curves, which represent sensitivity vs. 1 - specificity. The areas under the ROC curve were calculated, in which the values close to 1.0 indicate the highest diagnostic accuracy. The $95 \%$ confidence intervals were calculated for all coefficients. A $P$ value less than 0.05 was considered statistically significant. Statistical analysis was performed using SAS version 9.2 (SAS Institute).

\section{Results}

The data obtained from 135 subjects ( 78 men and 57 women; mean age, 48 years; range, 25-80 years), with neck pain (with $C R, n=67$; without $C R, n=68$ ) were analyzed. Demographic data are presented in Table 1. In the Spurling test, 37 of 67 patients with CR had positive results, and 67 of 68 patients without CR had negative results (Table 2). In the NTT, 57 of 67 patients with CR had positive results, and 59 of 68 patients without CR had negative results (Table 3).

Table 2. The Results of Spurling test

\begin{tabular}{llll}
\hline \multicolumn{5}{c}{ Spurling test } & \\
\hline CR & Positive & Negative & Total \\
Positive & 37 & 30 & 67 \\
Negative & 1 & 67 & 68 \\
Total & 38 & 97 & 135 \\
\hline \multicolumn{2}{l}{ CR: cervical radiculopathy. }
\end{tabular}

CR: cervical radiculopathy.

Table 3. The Results of NTT

\begin{tabular}{llll}
\hline \multicolumn{3}{l}{ NTT } & \\
\hline CR & Positive & Negative & Total \\
Positive & 57 & 10 & 67 \\
Negative & 9 & 59 & 68 \\
Total & 66 & 69 & 135 \\
\hline
\end{tabular}

NTT: neck tornado test; CR: cervical radiculopathy. 
The diagnostic discrimination of the Spurling test and the NTT are given in Table 4 . The Spurling test is superior in specificity (98.53 vs 86.76 ), PPV (97.37 vs 86.36 ), but NTT is superior in sensitivity (85.07 vs 55.22), accuracy (85.93 vs 77.04 ) and NPV (85.51 vs 69.07). The $P$ values of its sensitivity, specificity, PPV, NPV, and accuracy between the Spurling test and NTT were $<0.0001,0.0026,0.0075$, 0.0004 , and 0.0423 , respectively (Table 4 ).

The ROC curves were generated and used to compare these two tests (Figure 5).

Table 4. Comparison of the results of the Spurling test and the NTT.

\begin{tabular}{|c|c|c|c|}
\hline & The Spurling test & NTT & $P$ value \\
\hline AUC (95\% CI) & $76.88(70.71-83.05)$ & 85.92 (80.01-91.83) & $0.0252^{*}$ \\
\hline $\begin{array}{l}\text { Sensitivity (95\% } \\
\text { CI) }\end{array}$ & $55.22(43.32-67.13)$ & 85.07 (76.54-93.61) & $<0.0001^{*}$ \\
\hline $\begin{array}{l}\text { Specificity (95\% } \\
\text { CI) }\end{array}$ & 98.53 (95.67-101.39) & $86.76(78.71-94.82)$ & $0.0026^{*}$ \\
\hline $\begin{array}{l}\text { Accuracy }(95 \% \\
\text { CI) }\end{array}$ & $77.04(69.94-84.13)$ & $85.93(80.06-91.79)$ & $0.0423^{*}$ \\
\hline PPV $(95 \%$ CI $)$ & $97.37(92.28-102.46)$ & $86.36(78.08-94.64)$ & $0.0075^{*}$ \\
\hline NPV $(95 \%$ CI $)$ & 69.07 (59.87-78.27) & 85.51 (77.2-93.81) & $0.0004^{*}$ \\
\hline
\end{tabular}

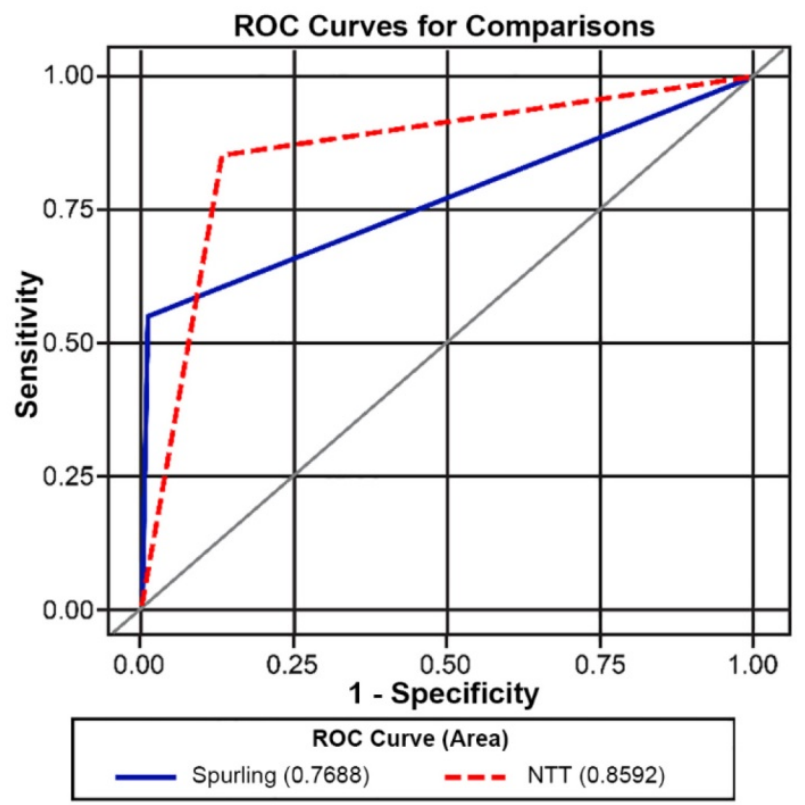

Figure 5. ROC curves of the Spurling test and the NTT. ROC: receiver operating characteristic; NTT: neck tornado test.

\section{Discussion}

We introduced a new provocative test, the NTT, to examine the neck and cervical spine in $\mathrm{CR}$ and to compare the diagnostic accuracy with a widely accepted provocative test, the Spurling test. The NTT yields high sensitivity and moderate to high specificity as a new screening test in CR. Overall, the diagnostic accuracy of the test appears to be superior compared with the Spurling test. The reason of higher accuracy of NTT compared to Spurling test is maybe that NTT is some high in both sensitivity and specificity. But Spurling test is higher in specificity but lower in sensitivity, so accuracy of Spurling test is lower than that of NTT.

While CR remains largely a clinical diagnosis, diagnostic imaging (myelogram, CT, or MRI) and electrophysiological studies (electromyography and nerve conduction studies) are commonly used to diagnose CR. Although these are considered the most accurate means of diagnosis available, each modality has inherent weaknesses: low cost/benefit ratio, discomfort, and often-lengthy waiting lists, with insufficient evidence to make a recommendation for or against the use of each diagnostic test [5, 12-15]. Asymptomatic radiological abnormalities are commonly seen in MRI, myelography, and CT of the cervical spine [16, 17]. The false-positive rate of imaging studies generally increases with advancing age and ranges from $20 \%$ to $50 \%$ [16, 18-20]. The evidence is insufficient to make a recommendation for or against the use of electromyography in patients in whom the diagnosis of $\mathrm{CR}$ is unclear after clinical examination and MRI [12, 21]. Therefore, a combination of clinical and radiological examinations should form the basis for the diagnosis of a significant root compression.

Physical examinations used in $\mathrm{CR}$ are the Spurling test (neck compression test), shoulder abduction (relief) sign, and neck distraction test, which have the following sensitivity and specificity: Spurling test, $40 \%-60 \%$ and $92 \%-100 \%$, respectively; shoulder abduction sign, $43 \%-50 \%$ and $80 \%-100 \%$, respectively; neck distraction test, $40 \%-43 \%$ and $80 \%-100 \%$, respectively. These tests have the characteristics of low sensitivity and high specificity [8].

The Spurling test, also known as the foraminal compression test, neck compression test, or the quadrant test, has been described as highly specific for cervical intraspinal pathologic lesions [9, 10]. A study by Shah and Rajshekhar [5] evaluated the test on 50 surgical patients with findings on MRI. The results of the study showed that the Spurling test was 92\% sensitive and $95 \%$ specific, with a PPV of $96.4 \%$ and an NPV of $90.9 \%$. Additional studies revealed that the Spurling test has a sensitivity of $40 \%-60 \%$ and specificity of $92 \%-100 \%$, concluding that the test has a high specificity but low sensitivity [10, 11]. When evaluating the correlation of a positive Spurling test 
with findings on electrophysiological studies, the Spurling test had a sensitivity of 30\% $(6 / 20)$ and a specificity of $93 \%(160 / 172)$ [9]. Our results were consistent with those of previous studies, demonstrating that although the Spurling test is not very sensitive (55.22\%), it is specific $(98.53 \%)$ for CR.

The Spurling test is designed to exacerbate the encroachment of exiting nerve roots by decreasing the dimensions of the foramen at a certain axis where compressive force is applied [6]. We speculated that this was the reason why the Spurling test yielded only low to moderate sensitivity (Figure 1 and 2) and designed a new physical examination that would supplement the weaknesses by applying compressive force while rotating the neck in a $180^{\circ}$ tornadic pattern (Figure 3). Because it is nearly impossible to pinpoint the exact location of the encroached exiting nerve root with the axis where compressive force should be applied to maximize the decrease in the dimension of the foramen, applying force at all angles appears to be a reasonable and an uncomplicated approach (Figure 4). Thus, the NTT is thought to be a simple and effective screening test in CR.

This study was limited by several factors. First, the patients were enrolled from just only one university hospital. The data may not be as accurate in a primary care clinic or other clinical settings. Second, different studies have used different criterion standards to diagnose $\mathrm{CR}$, and the diagnostic accuracy may vary accordingly. Tong et al. [9], using electrodiagnostic studies as a criterion standard in 224 patients, reported a sensitivity of $30 \%$ and specificity of $93 \%$ for the Spurling test. Uchihara et al. [22], using spinal cord deformity on MRI as the criterion standard in 65 patients, reported a sensitivity of $<28 \%$ and a specificity of $100 \%$ for the Spurling test. Our study used cervical disc herniation or other lesions that decrease the dimension of the foramen on MRI or $\mathrm{CT}$ as a criterion standard. When different criterion standards are used, the diagnostic accuracy of the NTT may subsequently be different from our results. Third, observer blinding was not applied when Spurling and NTT test were performed, i.e., the examiner knew the result of the Spurting test before the NTT test was undertaken. Fourth, reproducibility or interrater/intrarater reliability were not examined in this study due to retrospective research. Further study will be needed to increase interrater/intrarater reliability. Lastly, the NTT should be compared with various compression tests used to examine the cervical spine in CR, such as the Spurling test, as originally described by Spurling and Scoville [23], Jackson's neck compression test, in which the neck is flexed laterally then compressed [24, 25], and the neck compression test, in which the neck is only rotated then compressed [25]. Some clinicians also advocate that axial pressure should not be applied to the spine because this may be provocative and can exacerbate CR if present [9].

With the above limitations acknowledged, these data can serve as a rough guide on interpreting the Spurling test and the NTT in the clinical setting. The NTT is not as specific as the Spurling test, but it is a sensitive test with superior diagnostic accuracy for CR diagnosed by MRI. Therefore, the NTT can be useful clinically as a screening test and can help confirm CR along with the Spurling test.

\section{Abbreviations}

AUC: area under the curve; CI: confidence intervals; CR: cervical radiculopathy; CT: computed tomography; MRI: magnetic resonance imaging; NPV: negative predictive value; NTT: neck tornado test; PPV: positive predictive value; ROC: receiver operating characteristic.

\section{Acknowledgments}

The authors thank Dong-Su Jang (Research Assistant, Department of Anatomy, Yonsei University College of Medicine, Seoul, Korea) for his excellent support with the medical illustrations and Min Young Han (Assistant Manager, Division of Nursing, Severance Cardiovascular Hospital) for her excellent advice about management of research.

\section{Ethics Committee Approval}

This retrospective study was approved by the Institutional Review Board and the ethics committee of Gangnam Severance Hospital, Seoul, Korea (Approval No. 3-2015-0260).

\section{Patient Consent}

Consent was not required since the study was based on medical records of patients. The persons in the photographs (Figure 1 and 4) are first author Juyeon Park (green gown), and the corresponding author Jong Bum Choi (white gown) who are demonstrating the tests.

\section{Competing Interests}

The authors have declared that no competing interest exists.

\section{References}

1. Bono CM, Ghiselli G, Gilbert TJ, et al. An evidence-based clinical guideline for the diagnosis and treatment of cervical radiculopathy from degenerative disorders. Spine J. 2011; 11: 64-72.

2. Cohen I, Jouve C. Cervical radiculopathy. In: Frontera WR, Silver JK, Rizzo TD, eds. Essentials of physical medicine and rehabilitation : musculoskeletal disorders, pain, and rehabilitation, 2nd ed. Philadelphia, Pa: Saunders/Elsevier; 2008: 17-22. 
3. Radhakrishnan K, Litchy WJ, O'Fallon WM, et al. Epidemiology of cervical radiculopathy. A population-based study from Rochester, Minnesota, 1976 through 1990. Brain. 1994; 117 (Pt 2): 325-35.

4. De Luigi AJ, Fitzpatrick KF. Physical examination in radiculopathy. Phys Med Rehabil Clin N Am. 2011; 22: 7-40.

5. Shah KC, Rajshekhar V. Reliability of diagnosis of soft cervical disc prolapse using Spurling's test. Br J Neurosurg. 2004; 18: 480-3.

6. Corey DL, Comeau D. Cervical radiculopathy. Med Clin North Am. 2014; 98 : 791-9.

7. Malanga GA. The diagnosis and treatment of cervical radiculopathy. Med Sci Sports Exerc. 1997; 29: S236-45.

8. Malanga GA, Landes P, Nadler SF. Provocative tests in cervical spine examination: historical basis and scientific analyses. Pain Physician. 2003; 6: 199-205.

9. Tong HC, Haig AJ, Yamakawa K. The Spurling test and cervical radiculopathy. Spine (Phila Pa 1976). 2002; 27: 156-9.

10. Viikari-Juntura E, Porras M, Laasonen EM. Validity of clinical tests in the diagnosis of root compression in cervical disc disease. Spine (Phila Pa 1976). 1989; 14: 253-7.

11. Viikari-Juntura E. Interexaminer reliability of observations in physical examinations of the neck. Phys Ther. 1987; 67: 1526-32.

12. Ashkan $\mathrm{K}$, Johnston P, Moore AJ. A comparison of magnetic resonance imaging and neurophysiological studies in the assessment of cervical radiculopathy. Br J Neurosurg. 2002; 16: 146-8.

13. Landman JA, Hoffman JC, Jr., Braun IF, et al. Value of computed tomographic myelography in the recognition of cervical herniated disk. AJNR Am J Neuroradiol. 1984; 5: 391-4.

14. Van de Kelft E, van Vyve M. Diagnostic imaging algorithm for cervical soft disc herniation. J Neurol Neurosurg Psychiatry. 1994; 57: 724-8.

15. Yoss RE, Corbin KB, Maccarty CS, et al. Significance of symptoms and signs in localization of involved root in cervical disk protrusion. Neurology. 1957; 7: 673-83.

16. Boden SD, McCowin PR, Davis DO, et al. Abnormal magnetic-resonance scans of the cervical spine in asymptomatic subjects. A prospective investigation. J Bone Joint Surg Am. 1990; 72: 1178-84.

17. Wilson DW, Pezzuti RT, Place JN. Magnetic resonance imaging in the preoperative evaluation of cervical radiculopathy. Neurosurgery. 1991; 28: $175-9$.

18. Daniels DL, Grogan JP, Johansen JG, et al. Cervical radiculopathy: computed tomography and myelography compared. Radiology. 1984; 151: 109-13.

19. Jablecki CK, Andary MT, Di Benedetto M, et al. American Association of Electrodiagnostic Medicine guidelines for outcome studies in electrodiagnostic medicine. Muscle Nerve. 1996; 19: 1626-35.

20. Teresi LM, Lufkin RB, Reicher MA, et al. Asymptomatic degenerative disk disease and spondylosis of the cervical spine: MR imaging. Radiology. 1987; 164: 83-8.

21. Alrawi MF, Khalil NM, Mitchell P, et al. The value of neurophysiological and imaging studies in predicting outcome in the surgical treatment of cervical radiculopathy. Eur Spine J. 2007; 16: 495-500.

22. Uchihara T, Furukawa T, Tsukagoshi H. Compression of brachial plexus as a diagnostic test of cervical cord lesion. Spine (Phila Pa 1976). 1994; 19: 2170-3.

23. Spurling RG, Scoville WB. Lateral rupture of the cervical intervertebral discs: a common cause of shoulder and arm pain. Surg Gynecol Obstet. 1944; 78: 350-8.

24. Evans RC. Illustrated essentials in orthopedic physical assessment. St. Louis: Mosby; 1994.

25. Magee DJ. Orthopedic physical assessment. 6th ed. St. Louis, Missouri: Elsevier; 2014. 